\title{
EIGENFUNCTION CONCENTRATION FOR POLYGONAL BILLIARDS
}

\author{
ANDREW HASSELL, LUC HILLAIRET, AND JEREMY MARZUOLA
}

\begin{abstract}
In this note, we extend the results on eigenfunction concentration in billiards as proved by the third author in [8]. There, the methods developed in Burq-Zworski [2 to study eigenfunctions for billiards which have rectangular components were applied. Here we take an arbitrary polygonal billiard $B$ and show that eigenfunction mass cannot concentrate away from the vertices; in other words, given any neighbourhood $U$ of the vertices, there is a lower bound

$$
\int_{U}|u|^{2} \geq c \int_{B}|u|^{2}
$$
\end{abstract}

for some $c=c(U)>0$ and any eigenfunction $u$.

\section{INTRODUCTION}

Let $B$ be a plane polygonal domain, not necessarily convex. Let $V$ denote the set of vertices of $B$, and let $\Delta_{B}$ denote the Dirichlet or the Neumann Laplacian on $L^{2}(B)$. In this note, we will prove the following

Theorem 1. Let $B$ be as above and let $U$ be any neighbourhood of $V$. Then there exists $c=c(U)>0$ such that, for any $L^{2}$-normalized eigenfunction $u$ of the Dirichlet (or Neumann) Laplacian $\Delta_{B}$, we have

$$
\int_{U}|u|^{2} \geq c \text {. }
$$

That is, $U$ is a control region for $B$, in the terminology of [1].

We will generalize this result to any Euclidean surface with conical singularities (ESCS) $X$ with $U$ being any neighbourhood of the set of conical points and the $u_{k}$ being the eigenfunctions of the (Friedrichs)Laplace operator on $X$ (see Section 2 for more precise definitions).

Actually our main concern will be to derive a sufficient geometric condition for $U \subset X$ that ensures that $U$ is a control region for the Laplace operator. It is well-known that such a condition is obtained by the so-called geometric control (see [1] for instance) and we will be interested in regions $U$ for which geometric control fails. One major obstruction to geometric control is the existence of periodic orbits that do not intersect $U$. Since, on an ESCS, the non-singular periodic orbits form Euclidean cylinders immersed in $X$, we introduce the following geometric condition:

Definition 2. A region $U \subset X$ is said to satisfy condition $(C C)$ (the 'cylinder condition') if the following two properties hold.

(1) Any orbit that avoids $U$ is non-singular and periodic.

2000 Mathematics Subject Classification. 35P20.

Key words and phrases. Polygonal billiards, eigenfunction concentration, semiclassical measures, control region.

A.H. was partially supported by Discovery Grant DP0771826 from the Australian Research Council. J.M. was supported by a National Science Foundation Postdoctoral Fellowship and would like to thank Australian National University for generously hosting him at time of the beginning of this research. 


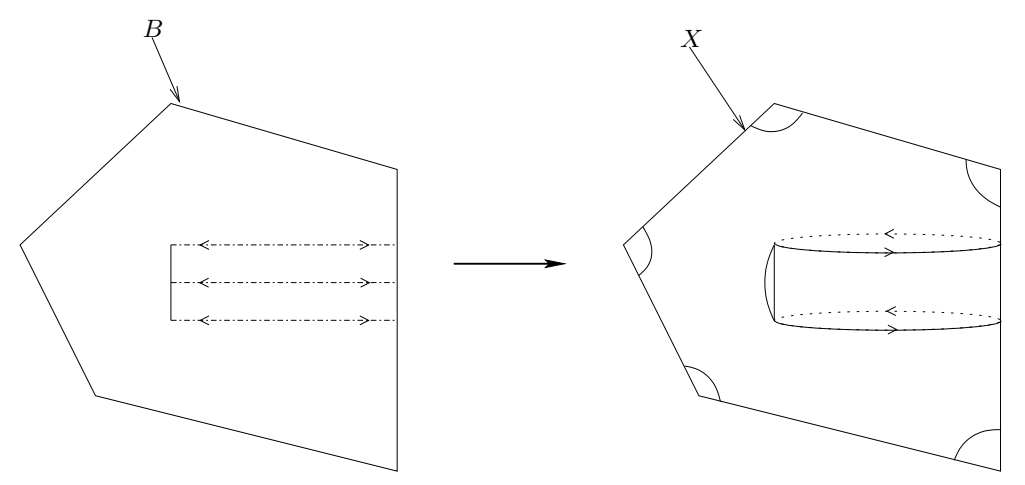

Figure 1. Doubling a billiard $B$, here with a slit, to form an ESCS $X$. Each vertex with angle $\alpha$ gives rise to a conic point with angle $2 \alpha$, and the endpoints of the slit become conic points with angle $4 \pi$.

(2) There exists a finite collection of cylinders $\left(\mathcal{C}_{i}\right)_{i \leq N}$ such that any orbit that avoids $U$ belongs to some $\mathcal{C}_{i}$.

Here, by a cylinder we mean an isometric immersion of $\mathbb{S}_{l}^{1} \times I$ into $X_{0}$, where $I \subset \mathbb{R}$ is an interval and $\mathbb{S}_{l}^{1}$ is the circle of length $l$ (see Lemma 7 below).

The main theorem of this paper is then the following.

Theorem 3. Let $X$ be an orientable ESCS and $U$ a domain satisfying $(C C)$. Then there exists a positive constant $c=c(U)$ such that any normalized eigenfunction $u_{k}$ of the Euclidean Laplace operator on $X$ satisfies

$$
\int_{U}\left|u_{k}\right|^{2} \geq c
$$

The first theorem is derived from this one by letting $X$ be the double of the polygon $B$ and by taking $U$ the $\varepsilon$ neighbourhood of the conical points of $X$, corresponding to the vertices of $B$; see Figure 1 The fact that, for billiards, $U$ satisfies $(C C)$ is the principal result of [3] (see Section 4 below).

Remark 4. Using Theorem[3, Theorem 1 can be sharpened by removing from $V$ those vertices with angle of the form $\pi / n$ for some integer $n$, as a reflection principle argument takes care of such vertices. Concretely, the geodesic flow may be non-ambiguously prolongated at such points. In particular, a cylinder hitting such a vertex on its boundary may be prolongated.

Remark 5. Let us mention other settings where our theorems apply. In the first theorem, the polygon may have polygonal holes and/or slits in it. In this case, $V$ should include the vertices of the holes and the ends of the slits. It also applies to translation surfaces, and thus also to tori with slits.

Remark 6. We will prove in Section 3 that any neighbourhood of the conical points satisfies condition $(C C)$ on any ESCS so that we could have stated the theorem without refering to this condition. We have stated it thus in order to emphasize the fact that such a control result usually requires two distinct steps. The first step is to find some geometric or dynamical condition that implies control, and the second step is to find settings where this condition holds. These two steps proceed from essentially different methods: analytic in the case of the first step, and geometric/dynamical in the case of the second. 
The organization of the paper reflects the two steps of the proof described in the preceding remark. We first recall in Section 2 some basic facts about ESCSs, semiclassical measures and the doubling procedure that allows one to treat polygonal billiards.

In Section 3, we will prove that $(C C)$ implies control. To do this, we shall argue by contradiction in the following way. Assume that there is no $c$ such that (1.1) holds. Then there is a sequence $\left(u_{n}\right)$ of normalized eigenfunctions with eigenvalues $\lambda_{n}^{2} \rightarrow \infty$, whose mass in $U$ tends to zero as $n \rightarrow \infty$. Associated to such a sequence is (at least one) semiclassical measure $\mu$ which is necessarily supported away from the inverse image $\pi^{-1}(U)$ - see Lemma 10. It is a standard property of such semiclassical measures that the support of $\mu$ is invariant under the billiard flow. We shall show that the support property of $\mu$ just mentioned and the geometric condition $(C C)$ imply that $\mu$ would have to be supported on the cylinders $\mathcal{C}_{i}$. So it suffices, for a contradiction, to show that its mass on each such cylinder is zero. To do this we use the argument of [8] (which in turn relies on [2]) slightly modified so as to avoid a technical assumption made there.

Finally, in Section 4, we demonstrate $(C C)$ for any neighbourhood of the set $P$ of conic points of an $\operatorname{ESCS} X$.

\section{ESCSS, POLYGONS AND SEMICLASSICAL MEASURES}

A Euclidean surface with conical singularities (ESCS) is a surface $X$ equipped with a metric $g$ such that $X$ may be written $X_{0} \cup P$ where the metric $g$ is Euclidean on $X_{0}$, and $P$ consists of a finite number of points $p_{i}$, such that each $p_{i}$ has a neighbourhood isometric to a Euclidean cone whose tip corresponds to $p_{i}$.

A reason for studying the Laplace operator on a ESCS is its relation with polygonal billiards. Indeed, starting from a polygon $B$, possibly with polygonal holes and/or slits, the following doubling procedure gives a ESCS $X$. Take two copies $B$ and $\sigma B$ of the polygon where $\sigma$ is a reflection of the plane. The double $X$ is obtained by considering the formal union $P \cup \sigma P$ where two corresponding sides are identified pointwise. The reflection $\sigma$ then gives an involution of $X$ that commutes with the Laplace operator. The latter thus decomposes into odd and even functions and the reduced operators are then equivalent to the Laplace operator in $P$ with Dirichlet and Neumann boundary condition respectively. In particular, for any $u_{n}$ eigenfunction of the Neumann, resp. Dirichlet Laplace operator in $P$, we can construct an eigenfunction of the Laplace operator in $X$ by taking $u$ in $P$ and $u \circ \sigma$, resp. $-u \circ \sigma$ in $\sigma P$.

On such a surface, we shall consider the geodesic flow induced by the Euclidean metric on $X_{0}$. We will not consider here the geodesics that end in a conical point since the condition $(C C)$ only considers non-singular geodesics. The following lemma shows that a non-singular periodic geodesic on a ESCS is always part of a family.

Lemma 7. Let $X$ be an orientable ESCS. Let $g: \mathbb{R} \rightarrow X$ be a non-singular T-periodic geodesic, then there exists $\delta>0$ such that $g$ extends to $h$ from $\mathbb{R} \times(-\delta, \delta)$ into $X_{0}$ such that

(1) $h(t, 0)=g(t)$

(2) $h$ is a local isometry from $\mathbb{R} \times(-\delta, \delta)$ equipped with the flat metric into $X_{0}$,

(3) $h$ is $T$-periodic in $t$.

Thus $h$ may be viewed as defined on the cylinder $\mathcal{C}_{\delta, T}:=\mathbb{S}_{T}^{1} \times(-\delta, \delta)$.

Proof. Let $T$ be the smallest period of $g$. For any $t \leq T$ there exists $\delta_{t}$ such that the square $\left(-\delta_{t}, \delta_{t}\right)^{2}$ is isometric to a neighbourhood of $g(t)$. Moreover, this isometry $h_{t}$ may be chosen so that the horizontal segment $\left(-h_{t}, h_{t}\right) \times\{0\}$ projects onto $g\left(t-h_{t}, t+h_{t}\right): h_{t}\left(t_{1}, 0\right)=\gamma\left(t+t_{1}\right)$. Using compactness, $\delta=$ $\inf \left\{\delta_{t}, t \in[0, T]\right\}$ exists and is positive. Gluing the $h_{t}$ by continuity defines a local isometry $h: \mathbb{R} \times(-\delta, \delta)$ into $X_{0}$. By construction, for any $s, h(t, s)$ is at distance $|s|$ of the geodesic $g$ and this distance is realized by 

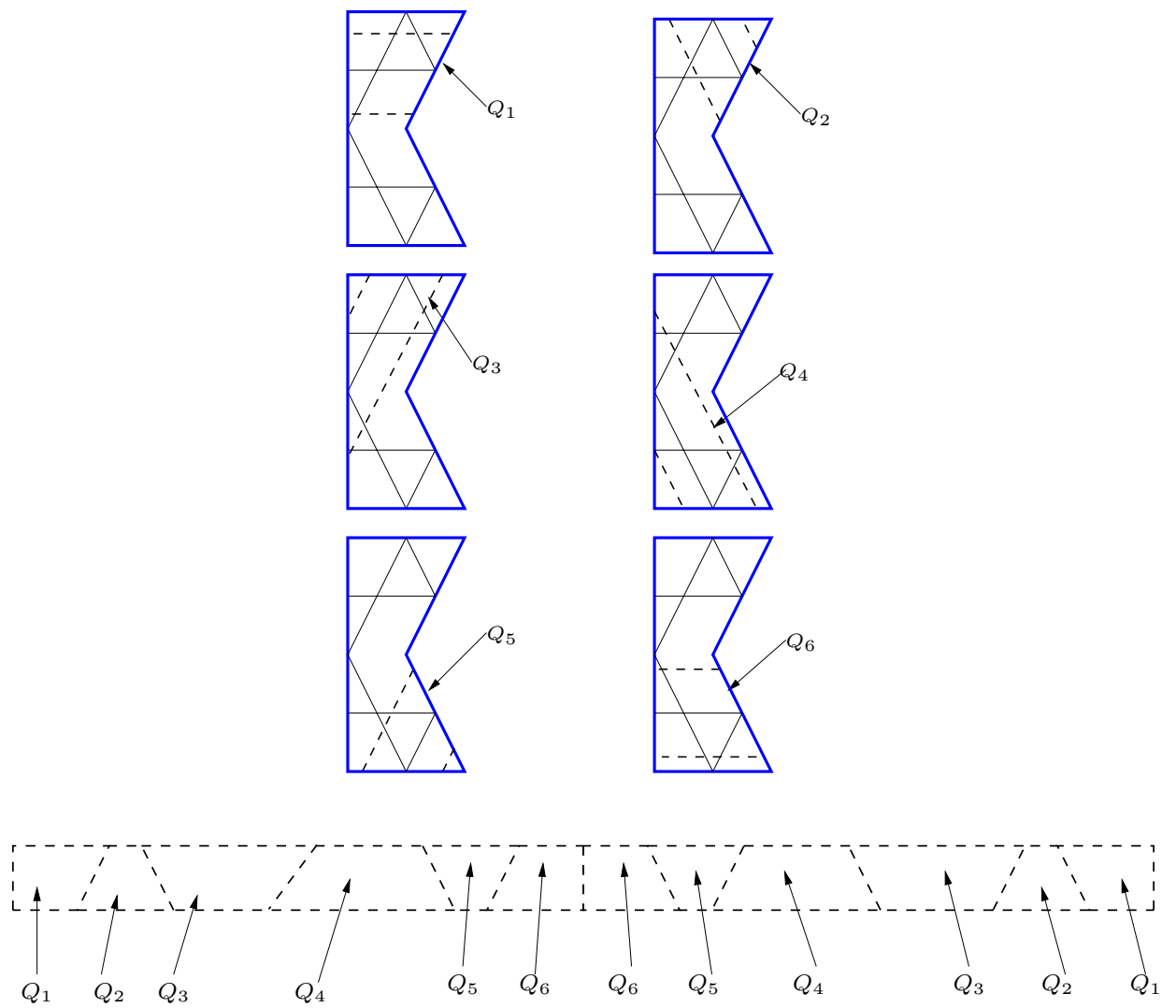

FiguRE 2. Construction of a cylinder from its components $Q_{j}$ along a periodic trajectory.

$g(t)$. Thus, there are only two possible choices for $h(t+T, s)$. Since $X$ is orientable, necessarily $h(t+T, s)=$ $h(t, s)$.

Let $\eta \in(-\delta, \delta)$. As $\eta \uparrow \delta$, the periodic geodesics $h(t, \eta)$ converge to a possibly singular periodic geodesic (and similarly for $\eta \downarrow-\delta$ ). The cylinder $\mathcal{C}_{\delta, T}$ will be called maximal if both these geodesics are singular. In the geometric condition $(C C)$, we may assume that the cylinders are maximal.

We now define the Euclidean Laplace operator on a ESCS. First note that the Euclidean metric on $X$ provides us with a well-defined $L^{2}$ norm and that smooth functions compactly supported in $X_{0}$ are dense in $L^{2}(X)$. For any such function, we can also define the quadratic form $q(u)=\int_{X}|\nabla u|^{2} d x$ in which $\nabla$ is taken with respect to the Euclidean metric and $d x$ is the Euclidean area element. The Laplace operator is the self-adjoint operator associated with the closure of this quadratic form. It is also the Friedrichs extension of the usual Euclidean Laplace operator defined on $\mathcal{C}_{0}^{\infty}\left(X_{0}\right)$. It is standard that this operator has compact resolvent so that its spectrum is purely discrete and we may consider its eigenvalues and eigenfunctions.

Let $u_{n}$ be a sequence of eigenfunctions on $X$ associated with a sequence of eigenvalues going to infinity. We want to associate to this sequence a so-called semiclassical measure. Since we do not want to look precisely at what is happening at the conical point, our semiclassical measure $\mu$ will be a positive distribution acting on $\mathcal{C}_{0}^{\infty}\left(S^{*} X_{0}\right)$, where $S^{*} X_{0}$ denotes the unit cotangent bundle over $X_{0}$. Our semiclassical measure is then given by the usual recipe. In particular, for any $a \in \mathcal{C}_{0}^{\infty}\left(S^{*} X_{0}\right)$ and any zeroth-order 
pseudodifferential operator $A$ on $X$ with principal symbol $a$ we have

$$
\lim _{n \rightarrow \infty}\left\langle A u_{n}, u_{n}\right\rangle=\int_{S^{*} X_{0}} a d \mu .
$$

Remark 8. It is considerably simpler to define a pseudodifferential operator on $X_{0}$ than on $X$. In particular we may use local isometries with the Euclidean plane.

Remark 9. It should be noted that, in contrast with the usual semiclassical measure, with this definition, a semiclassical measure need not be a probability measure. In order to be a probability measure one has to prove that, loosely speaking, no mass accumulates at the conical points.

The invariance property of this measure by the geodesic flow also has to be taken carefully. The infinitesimal version of this invariance is true using the standard commutator argument and Egorov's theorem since this computation takes place integrally over $X_{0}$. One can then integrate this property along any geodesic until it reaches a vertex.

\section{Proof of the main theorem}

Let $U$ be a domain of $X$ satisfying condition $(C C)$. Necessarily we have $P \subset U$. We will denote by $U_{0}=U \backslash P$. Let $u_{n}$ be a sequence of normalized eigenfunctions such that $\int_{U}\left|u_{n}\right|^{2} \rightarrow 0$. Let $\mu$ be any semiclassical measure associated to $\left(u_{n}\right)$. Then we have

Lemma 10. (i) The support of $\mu$ is disjoint from $\pi^{-1}\left(U_{0}\right)$.

(ii) $\mu$ is a probability measure that is invariant under the geodesic flow.

Proof. (i) Suppose that there is a point $q \in \operatorname{supp} \mu$ with $\pi(q) \in U_{0}$. Choose a nonnegative function $\phi \in C^{\infty}\left(X_{0}\right)$ supported in $U_{0}$, with $\phi \equiv 1$ in a small neighbourhood $G$ of $\pi(q)$. Since $\phi \geq 0$ and $\mu$ is a positive measure, we have $\langle\mu, \phi\rangle \geq 0$. If $\langle\mu, \phi\rangle=0$ then $\langle\mu, \chi\rangle=0$ for every $\chi \in C_{0}^{\infty}\left(S^{*} X_{0}\right)$ supported in $\pi^{-1}(G)$, since we have $\chi=\chi \phi$, and by the positivity of $\mu$ and $\phi,|\langle\mu, \chi \phi\rangle|$ is bounded by $\langle\mu, \phi\rangle\|\chi\|_{\infty}$. But this would mean that $\pi^{-1}(G)$ is disjoint from the support of $\mu$, which is not the case. Thus we conclude that $\langle\mu, \phi\rangle>0$. This means that

$$
\lim _{n \rightarrow \infty} \int_{B}\left|u_{n}\right|^{2} \phi>0,
$$

contradicting our assumption about the sequence $\left(u_{n}\right)$. This proves (i).

(ii) Statement (i) tells us precisely that no mass accumulates at the conical points and thus $\mu$ is a probability measure (see Remark 9 above). The invariance holds since $\mu$ is a semiclassical measure.

Let $\mu$ be as above, and let $(z, \zeta) \in T^{*} X_{0}$ be in the support of $\mu$. According to the preceding lemma and the invariance property of $\mu$, condition $(C C)$ implies that $z$ belongs to a cylinder periodic in the direction $\zeta$.

The support of $\mu$ is thus included in the union of the maximal cylinders $\mathcal{C}_{i}$ defined in condition $(C C)$.

Let $\mathcal{C}$ be such a cylinder. By definition, there is a local isometry between $\mathbb{S}_{L}^{1} \times(0, a)$ and $\mathcal{C}$. Using it, we can pull-back the eigenfunction $u_{n}$ to $\mathcal{C}$. We now apply the argument of [8] to this function $u_{n}$ on $\mathcal{C}$. Let us use Cartesian coordinates $(x, y)$ on $\mathcal{C}$, where $x \in[0, L], y \in[0, a]$ with $x=0$ and $x=L$ identified. Thus $\{y=0\}$ and $\{y=a\}$ are the two long sides of the cylinder, and the variable $y$ parametrizes periodic geodesics. Choose a cutoff function $\chi \in C_{c}^{\infty}[0, a]$ such that $\chi=1$ on an open set containing all $y$ parametrizing all paths disjoint from $U_{\epsilon}$ (as opposed to $U_{\epsilon / 2}$ ). Then $\chi u_{n}$ vanishes near the long sides of $\mathcal{C}$, and thus may be regarded as a function on a torus $T$. So we now have a sequence $v_{n}=\chi u_{n}$ on $T$. Consider any semiclassical measure $\nu$ associated with the sequence $\left(v_{n}\right)$ on $T$. By compactness, the $v_{n}$ are 
bounded in $L^{2}$, so there exists at least one semiclassical measure associated with $\left(v_{n}\right)$ (on the torus). This could be the zero measure; this would be the case if $\left\|v_{n}\right\|_{L^{2}} \rightarrow 0$, for example. Since $\mu$ is supported on a finite number of cylinders, there are only a finite number of directions in the support of $\nu$. So we can find a constant-coefficient pseudodifferential operator $\Phi$ on $T$ that is microlocally 1 in a neighbourhood of directions parallel to $d x$, i.e. in the direction of the unwrapped periodic paths, but vanishes microlocally in a neighbourhood of every other direction in the support of $\nu$. (See [8] for a discussion of constant-coefficient pseudodifferential operators on a torus.)

Consider the sequence of functions $\left(\Phi v_{n}\right)$ on $T$. The semiclassical measures $\nu^{\prime}$ associated to this sequence are related to those for the sequence $\left(v_{n}\right)$ by $\nu^{\prime}=\sigma(\Phi) \nu$. Thus, the support of $\nu^{\prime}$ is restricted to directions parallel to $d x$ and to geodesics parametrized by $y$ such that $\chi(y)=1$ (because of lemma 10 and the way we chose $\chi)$.

Now we apply the proposition on p46 of [2] which says:

Proposition 11. Let $\Delta=-\left(\partial_{x}^{2}+\partial_{y}^{2}\right)$ be the Laplacian on a rectangle $R=[0, l]_{x} \times[0, a]_{y}$. For any open $\omega \subset R^{2}$ of the form $[0, l]_{x} \times \omega_{y}$, there is $C$ such that, for any solution of

$$
\left(\Delta-\lambda^{2}\right) w=f+\partial_{x} g
$$

on $R$, satisfying periodic boundary conditions, we have

$$
\|w\|_{L^{2}(R)}^{2} \leq C\left(\|f\|_{L^{2}(R)}^{2}+\|g\|_{L^{2}(R)}^{2}+\|w\|_{L^{2}(\omega)}^{2}\right) .
$$

(This proposition is stated in [2] for Dirichlet boundary conditions on a rectangle, but applies equally well to periodic boundary conditions as noted in [8].) We apply this with $w=w_{n}=\Phi v_{n}, f=f_{n}=\Phi\left(\left(\partial_{y}^{2} \chi\right) u_{n}\right)$, $g=g_{n}=-2 \Phi\left(\left(\partial_{y} \chi\right) u_{n}\right)$, and $\omega$ contained in the set $\{\chi=0\}$. (Note that $\Phi$ commutes with $\Delta$ and $\partial_{y}$.) Since $f$ and $g$ are supported on the support of $\nabla \chi$, their support is disjoint from that of $\nu^{\prime}$, so $\left\|f_{n}\right\|_{L^{2}(R)}^{2}+\left\|g_{n}\right\|_{L^{2}(R)}^{2} \rightarrow 0$ as $n \rightarrow \infty$. Also, by our choice of $\omega$, we have $\left\|w_{n}\right\|_{L^{2}(\omega)}^{2}=0$. It follows that $\left\|w_{n}\right\|_{L^{2}(R)}^{2} \rightarrow 0$. But this means that $\nu^{\prime}=0$. This implies that $\nu$ has no mass along directions parallel to $d x$, which means that $\mu$ has no mass along the cylinder $\mathcal{C}$. Since $\mathcal{C}$ is arbitrary, and the number of such cylinders is finite, this means that $\mu$ has no mass, i.e. it is the zero measure. This contradicts part (ii) of Lemma 10, We conclude that Theorem 3 holds.

\section{Condition $(C C)$ For neighbourhoods of the Conic set $P$}

In this section we study condition $(C C)$ in more detail. We wish in particular to address this condition for the region $U_{\epsilon}$ which is the $\varepsilon$ neighbourhood of the set $P$ of conical points of an ESCS $X$.

In this case we have the following proposition.

Proposition 12. Let $X$ be an orientable ESCS with singular set $P$.

(i) For any geodesic $\gamma$, either $\gamma$ is periodic, or the closure of $\gamma$ meets $P$.

(ii) Let $U_{\epsilon}$ denote the $\varepsilon$ neighbourhood of $P$. Then any periodic geodesic avoiding $U_{\varepsilon}$ (which is periodic by part (i)) belongs to a maximal cylinder and the number of such maximal cylinders is finite.

Before proving Proposition [12, we introduce some notation and definitions. A strip is an isometric immersion $h: \mathbb{R} \times I \rightarrow X_{0}$, where $I$ is a nonempty open interval and $\mathbb{R} \times I$ is equipped with the Euclidean metric. We will also sometimes call the image of $h$ a strip. The width of the strip is the length of the interval $I$.

For any strip, the mappings $\gamma_{c}:=h(\cdot, c), c \in I$, are geodesics of $X_{0}$. Since $h$ is a local isometry and $X$ is orientable, if one $\gamma_{c}$ is periodic of length $L$ then, for any $c^{\prime}, \gamma_{c^{\prime}}$ is also periodic with length $L$. 
A maximal strip is a strip that cannot be extended to $\mathbb{R} \times I^{\prime}$ for any open $I^{\prime}$ properly containing $I$. A strip is maximal if and only if $P$ intersects the closure of $h(\mathbb{R} \times I)$ on its left and on its right.

For any geodesic $\gamma$ we will denote by $\tilde{\gamma}$ the geodesic lifted to the unit tangent bundle $S X_{0}$ and we denote by $\pi$ the projection of $S X_{0}$ in $X$. We also denote by $d(.,$.$) the distance on X$.

Proposition 12 is a straightforward consequence of the following lemma, which is closely related to results of [5].

Lemma 13. Let $h: \mathbb{R} \times I \rightarrow X_{0}$ be a strip of positive width. Then there exists $L$ such that $h(t+L, s)=$ $h(t, s)$.

Proof. We follow closely the ideas of [5]. We may assume that $h$ is maximal and $I=(-\delta, \delta)$ and we will prove that $\gamma_{0}$ is periodic. Observe that for any $t, d\left(\gamma_{0}(t), P\right) \geq \delta$. We denote by $Z \subset S X_{0}$ the forward limit set of the lifted geodesic $\tilde{\gamma}_{0}$. By continuity, we have that $d(\pi(Z), P) \geq \delta$. This implies first that $Z$ is compact and then that the geodesic flow is continuous on $Z$. Using Furstenberg's uniform recurrence theorem [4], there exists a point $x$ that is uniformly recurrent in $Z$. We denote by $G$ the geodesic emanating from $x$ (observe that $\tilde{G}(\mathbb{R}) \subset Z$ ). We also denote by $H: \mathbb{R} \times\left(-\Delta^{-}, \Delta^{+}\right)$the maximal strip around $G$. Uniform recurrence means the following: for any neighbourhood $\tilde{W} \subset S M_{0}$ that intersects $\tilde{G}(\mathbb{R})$, there exists $L \in \mathbb{R}$ such that

$$
\forall t, \exists s \in[t, t+L] \text { such that }(G(s), \dot{G}(s)) \in \tilde{W} .
$$

The uniform recurrence and the maximality of the strip imply

$$
\forall \varepsilon>0, \exists L \text { such that } \forall t, d\left(H\left([t, t+L] \times\left\{\Delta^{+}-\varepsilon\right\}\right), P\right)<2 \varepsilon .
$$

Indeed, by maximality, for any $\varepsilon>0$, there exists $t_{0}$ such that the geodesic $\bar{\gamma}$ emanating from the point in phase space given by $\left(G\left(t_{0}\right), \dot{G}\left(t_{0}\right)-\frac{\pi}{2}\right)$ hits a conical point in time less than $\Delta^{+}+\frac{\varepsilon}{2}$. In particular

$$
d\left(H\left(t_{0}, \Delta^{+}-\varepsilon\right), P\right) \leq \frac{3 \varepsilon}{2} .
$$

By continuity, we can find a neighbourhood $\tilde{V}$ of $\left(G\left(t_{0}\right), \dot{G}\left(t_{0}\right)\right)$ such that, for any $(m, \theta)$ in this neighbourhood, the geodesic starting from $\left(m, \theta-\frac{\pi}{2}\right)$ stays in the $\frac{\varepsilon}{2}$ tubular neighbourhood of $\bar{\gamma}$ until time $\Delta^{+}-\varepsilon$. Using uniform recurrence, there exists $L$ such that, for any $t$, there exists $s \in[t, t+L]$ so that $(G(s), \dot{G}(s))$ belongs to $\tilde{V}$. Using the preceding property we have that

$$
d\left(H\left(s, \Delta^{+}-\varepsilon\right), H\left(t_{0}, \Delta^{+}-\varepsilon\right)\right)<\frac{\varepsilon}{2} .
$$

We conclude (4.1) using the triangle inequality.

Observe that (4.1) means that if we represent the strip $H$ by a vertical strip in $\mathbb{R}^{2}$ then we can find a vertical strip of width $2 \varepsilon$ (that contains the right boundary of the strip $H$ ) such that any rectangle of height $L$ contained in this strip contains at least one conical point.

We fix local coordinates near $x$ so that $x=\left((0,0), \frac{\pi}{2}\right)$. Since $x$ is in the forward limit set, there exists $t_{n}$ such that $\tilde{\gamma}_{0}\left(t_{n}\right)$ converges to $x$. We set $\tilde{\gamma}_{0}\left(t_{n}\right)=\left(z_{n}, \theta_{n}\right)$. Represent now in $\mathbb{R}^{2}$, the strip $H$ around $(0,0)$ and the strip $h$ around $z_{n}$. By standard Euclidean geometry, for $\epsilon<<\delta$ the intersection of any vertical strip of width $2 \varepsilon$ with $h$ contains a vertical rectangle of width $2 \varepsilon$ and height that goes to $\infty$ when $\theta$ goes to $\frac{\pi}{2}$ (see Figure 3). Indeed, denoting by $\alpha=\frac{\pi}{2}-\theta$, this height is

$$
\frac{2 \delta}{\sin |\alpha|}-\frac{2 \varepsilon}{\tan |\alpha|} \text {. }
$$

Using (4.1), since there is no conical point in $h$, this implies that we have $\theta_{n}=\frac{\pi}{2}$ for $n$ large enough. The strip $h$ around $z_{n}$ is thus represented by a vertical strip of width $2 \delta$. Then maximality of both $h$ and $H$ 


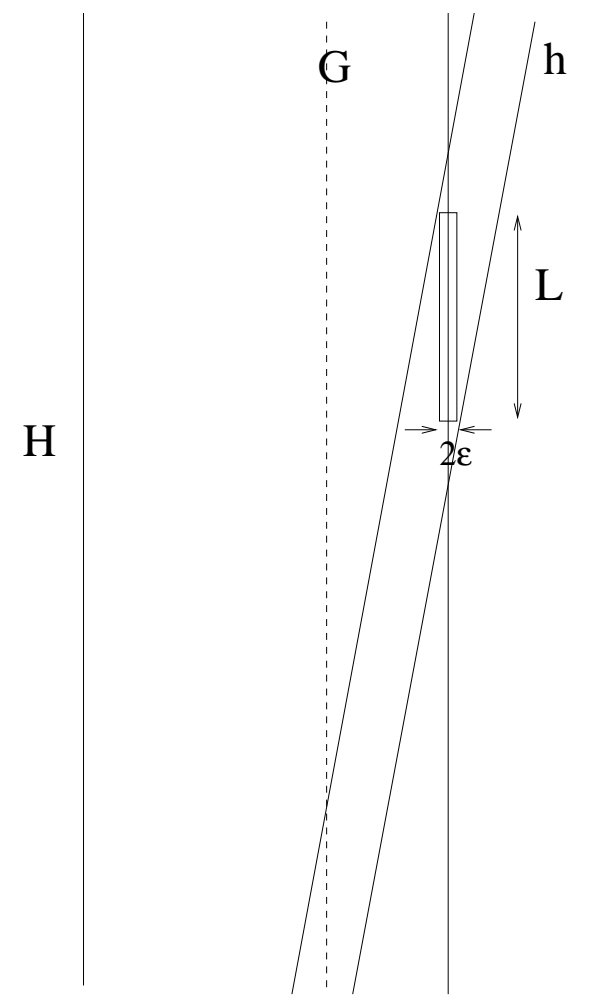

FiguRE 3. Illustration of the argument in the proof of Lemma 13. For sufficiently large $n$, if $\theta_{n} \neq \pi / 2$ then the strip $h$ would contain a rectangle of size $2 \epsilon \times L$, as illustrated. This is not possible as any such rectangle intersects $P$.

implies that the strips coincide up to a translation in the first variable; in particular, the widths coincide, and $z_{n}$ is independent of $n$. But this implies that the geodesic $\gamma_{0}$ is periodic.

Proof of Proposition 12, (i) Let us consider a geodesic $g$ such that $g(\overline{\mathbb{R}})$ contains no conical point. There exists $\epsilon>0$ such that $\forall t, B(g(t), \epsilon) \cap P=\emptyset$. For, otherwise, we could find sequences $\epsilon_{n}, t_{n}$, and $p_{n}$ such that $d\left(g\left(t_{n}\right), p_{n}\right)<\epsilon_{n}$ contradicting the hypothesis. This implies that the geodesic $g: \mathbb{R} \rightarrow X_{0}$ extends to a strip of positive width and Lemma 13 concludes the proof.

(ii) We only have to prove the finiteness property. Denote by $\mathcal{C}_{i}$ the maximal cylinders. By definition the middle geodesic of $\mathcal{C}_{i}$ is at distance at least $\varepsilon$ of the conical points. So that the $\varepsilon / 2$ strip around this geodesic consists in periodic geodesics at distance at least $\varepsilon / 2$ of the conical points. Denote by $S_{i}$ this strip. The proof of Lemma 4.2 of [7] (see also fig. 2 of this reference [3]) implies that if $\gamma_{i}$ and $\gamma_{j}$ are two periodic geodesics in strip $S_{i}$ and $S_{j}$ respectively then, at any of their intersections, they make an angle $\theta$ satisfying

$$
\frac{1}{\sin \theta} \leq \frac{\min \left(L_{i}, L_{j}\right)}{\varepsilon} .
$$

We can now adapt the argument of [3]. Fo any $i$ we consider the following region $V_{i}$ of $S X_{0}$,

$$
V_{i}=\left\{(x, \theta), x \in S_{i},\left|\theta-\theta_{i}\right|<\frac{\varepsilon}{2 L_{i}}\right\} .
$$


The preceding estimate implies :

(1) $V_{i}$ is isometric to $(-\varepsilon / 2, \varepsilon / 2) \times \mathbb{S}_{L_{i}} \times\left(-\frac{\varepsilon}{2 L_{i}}, \frac{\varepsilon}{2 L_{i}}\right)$,

(2) for different cylinders, the regions $V_{i}$ are distinct.

The first point implies that the volume of $V_{i}$ is bounded away from zero independently of the cylinder, and the second point coupled with the fact that the unit tangent space to $X$ has finite volume yields the result.

Remark 14. We have seen that the $\varepsilon$ neighbourhood $U_{\epsilon}$ of the conical points is a control region for $X_{0}$. Since any geodesic that enters the $\varepsilon$-neighbourhood also enters the annular region $\varepsilon / 2 \leq d(x, P) \leq \varepsilon$, the union of these annular regions is also a control region. A similar argument also shows that for any $\delta>0$ and any $\varepsilon>0$ the $\varepsilon$-neighbourhood of the union of the normal co-bundle to the circles $d(x, p)=\delta$ is a control region (in the cotangent bundle!).

\section{REFERENCES}

[1] N. Burq and M. Zworski. Geometric control in the presence of a black box. Jour. Amer. Math. Soc., 17(2), 443-471, 2004.

[2] N. Burq and M. Zworski. Bouncing ball modes and quantum chaos. SIAM Review, 47(5), 43-49, 2005.

[3] C. I. Delman, G. Galperin, Billiards with pockets: a separation principle and bound for the number of orbit types, Comm. Math. Phys. 230(3), 463-483, 2002.

[4] H. Furstenberg, Recurrence in ergodic theory and combinatorial number theory, Princeton University Press, Princeton, 1981.

[5] G. Galperin, T. Krüger, S. Troubetzkoy, Local instability of orbits in polygonal and polyhedral billiards, Comm. Math. Phys. 169(3), 463-473, 1995

[6] P. Gérard and E. Leichtnam. Ergodic properties of eigenfunctions for the Dirichlet problem. Duke Math. J., 71(2), 559-607, 1993.

[7] L. Hillairet, Clustering of eigenvalues on translation surfaces. Ann. Henri Poincaré, 7(4), 689-710, 2006.

[8] J. Marzuola. Eigenfunctions for partially rectangular billiards, Communications in Partial Differential Equations, 31, 775-790, 2007.

[9] S. Zelditch and M. Zworski. Ergodicity of Eigenfunctions for Ergodic Billiards, Commun. Math. Phys., 175, 673-682, 1996.

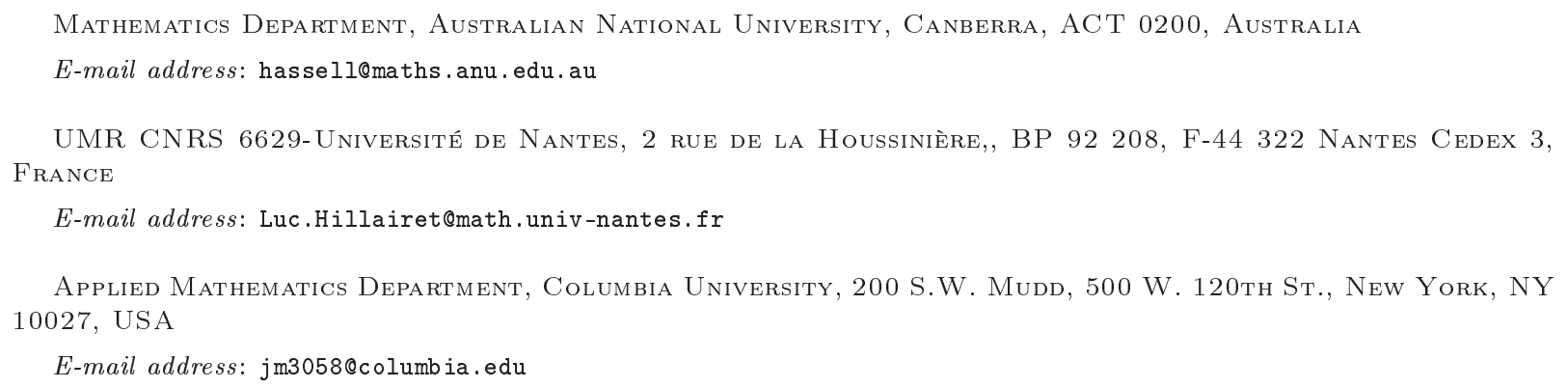

E-mail address: jm3058@columbia.edu 